\title{
A Survey of Camera Self-Calibration
}

\author{
Elsayed E. Hemayed \\ Computer Science and Eng. Dept, Faculty of Engineering, Cairo University, Egypt \\ hemayed@ieee.org
}

\begin{abstract}
This paper surveys the developments of the last 10 years in the area of camera self-calibration. In order to solve this problem, researches have used the camera intrinsic constraints separately and in conjunction with the camera motion constraints or the scene constraints. Most of the self-calibration algorithms are concerned with unknown but constant intrinsic camera parameters. Recently, camera self-calibration in the case of varying intrinsic camera parameters was also studied. We present the basic theories behind the different self-calibration techniques and discuss the ideas behind most of the self-calibration algorithms.
\end{abstract}

\section{Introduction}

Self-Calibration, introduced by $[1,2]$, is an attempt to calibrate the camera(s) by finding intrinsic parameters that are consistent with the underlying projective geometry of a sequence of images. In doing that, the self-calibration algorithms make no or few assumptions about the particular structure of the scene being viewed. In this survey, we'll call the underlying projective geometry constraints, the camera intrinsic constraints.

Recently, researchers extend the self-calibration techniques to use other constraints besides the camera intrinsic constrains, mainly the camera motion constraints and the scene constraints. The dimension of the selfcalibration problem has been extended also to address varying intrinsic parameters, mainly the zoom and the focus of the camera. A key use of such a system is as a solution to the registration problem of augmented reality; the estimation of the alignment between the real and virtual world [3].

This paper divided into eight sections including the introduction section. Section 2 addresses the basic principles that most of the self-calibration techniques rely on. Sections 3-6 address the different classes of selfcalibration techniques. Section 3 addresses the techniques that rely solely in the camera intrinsic constraints. Section 4 addresses the techniques that combine the camera motion and the camera intrinsic constraints to solve the problem. Section 6 addresses the techniques that combine the scene constraints and the camera intrinsic constraints to solve the calibration problem. Section 6 addresses the critical motion sequences. Section 7 concludes the survey and finally Section 8 list the citations referenced in this paper.

\section{Basic principles}

The theoretical foundations of the self-calibration techniques are presented in this section. Starting first with the projective geometry of the camera model (pinhole camera model is used). Then, introducing the concept of the absolute conic and its usage in developing the basis for the self-calibration techniques.

\subsection{Projective geometry}

In the pinhole camera model, the camera is assumed to perform a perfect perspective transformation. Let $(\mathrm{u}, \mathrm{v})$ be the image coordinates of the world coordinates $(\mathrm{X}, \mathrm{Y}, \mathrm{Z})$ then, the equation of the projection is

$$
\left[\begin{array}{l}
s u \\
s v \\
s
\end{array}\right]=\mathrm{K} R^{\mathrm{T}}[\mathrm{I} \mid-t]\left[\begin{array}{l}
X \\
Y \\
Z \\
1
\end{array}\right]=\mathrm{P}\left[\begin{array}{l}
X \\
Y \\
Z \\
1
\end{array}\right]
$$

Where $\mathrm{K}$ is an upper triangle matrix accounting for the intrinsic camera parameters and $R$ and $t$ is accounting for camera orientation and position, respectively, that is the extrinsic camera parameters. The matrix $\mathrm{P}$ is the perspective transformation matrix, which relates 3D world coordinates and 2D image coordinates. The matrix $\mathrm{K}$ depends on a variable number of parameters, according to the sophistication of the camera model. In our case, there are five intrinsic parameters as shown in Equation 2 where $\left(u_{0}, v_{0}\right)$ is the coordinates of the principle point, $\left(\alpha_{v}, \alpha_{v}\right)$ are scale factors, and $\theta$ is the angle between the image axes.

$$
K=\left[\begin{array}{ccc}
\alpha_{u} & -\alpha_{u} \cot (\theta) & u_{0} \\
0 & \alpha_{v} / \sin (\theta) & v_{0} \\
0 & 0 & 1
\end{array}\right]
$$

\subsection{Absolute conic and Kruppa equations}

The absolute conic $\Omega$ is a particular conic in the plane at infinity. The conic $\Omega$ is invariant under rigid motions and under uniform changes of scale so its relative position to a moving camera is constant. Therefore its image $\omega$ will be constant if the intrinsic camera parameter is constant. 
The conic $\Omega$ can be considered a calibration object that is present in all scenes.

The conic $\Omega$ can be represented by the Dual Absolute Quadric $\Omega$ '. In this case, both $\Omega$ and its supporting plane, the plane at infinity $\Pi$, are expressed through one geometric entity and the relationship between the $\Omega$ and its image $\omega$ is easily obtained using the projection equation for the $\Omega$ ':

$$
\omega_{i}^{\prime} \approx \mathrm{P}_{i} \Omega^{\prime} \mathrm{P}_{i}^{\mathrm{T}}
$$

Where the operator $\approx$ means up to a scale factor, $\omega_{i}^{\prime}$ represents the dual of $\omega_{i}, \Omega$ the dual of $\Omega$ and $P_{i}$ the projection matrix for view $i$ (see Fig. 1). Using Equations 1 and 3 and writing $\Omega$ ' as $=\operatorname{diag}(1,1,1,0)$, one obtains the relation between the intrinsic camera parameters $\mathrm{K}$ and the dual of the image of the absolute conic $\Omega$ ':

$$
\omega_{i}^{\prime} \approx \mathrm{K}_{i} \mathrm{~K}_{i}^{\mathrm{T}}
$$

The $\Omega$ can be transferred from one image to another through the homography of its plane (i.e. the plane at infinity):

$$
\omega_{j} \approx H_{i j}^{\infty-\mathrm{T}} \omega_{i} H_{i j}^{\infty-1}
$$

Then if we restrict this constraint to the epipolar geometry, one obtains the Kruppa equations [4] (see Fig. 1):

$$
\left[e_{i j}\right]_{x}^{\mathrm{T}} \mathrm{KK}^{\mathrm{T}}\left[e_{i j}\right]_{x} \approx F_{i j} \mathrm{KK}^{\mathrm{T}} F_{i j}^{\mathrm{T}}
$$

where $F_{i j}$ the fundamental matrix for views $i$ and $j$, and $\mathrm{e}_{\mathrm{ij}}$ the corresponding epipole. By this restriction we eliminate the position of infinity from the equation. In this case only 2 independent equations can be obtained. Combining Equation (5) and (6) one obtain the following equation:

$$
\mathrm{K}_{i} \mathrm{~K}_{i}^{\mathrm{T}} \approx \mathrm{P}_{i} \Omega^{\prime} \mathrm{P}_{i}^{\mathrm{T}}
$$

Equations 5-7 are the foundations for the majority of the camera self-calibration techniques. When the homography of the plane at infinity $H_{i j}^{\infty}$ is known, then Equation (5) can be reduced to a set of linear equations in the coefficients of $\omega_{i}$ or $\omega_{i}^{\prime}$ [5].

In the next sections, we are going to present the different techniques of camera self-calibration grouped according to the used constraints. Each section will have two subsections, one for constant intrinsic and the other for the varying intrinsic case.

\section{Camera intrinsic constraints}

Many researchers have been working on self-calibration subject. Mostly self-calibration algorithms are concerned with unknown but constant intrinsic camera parameters. The techniques presented in this section rely only on the

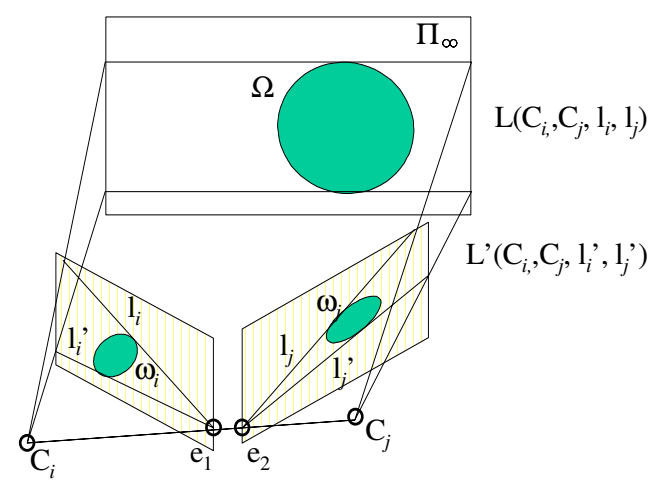

Figure 1. The image of the absolute conic
satisfies the epipolar constraint.

camera intrinsic constraints and had no assumptions about the particular structure of the scene being viewed. Some interesting approaches were proposed in[1, 2, 4-23, 27-29].

\subsection{Constant intrinsic}

The first self-calibration method was proposed in [1, 2] based on the Kruppa equations (Equation 6) when up to three views of a scene are available. The approach was improved over the years [6]. Luong and Faugeras [6] use the kruppa equations to derive systems of polynomial equations, which are of degree four in five unknowns. These systems are solved with the use of numerical continuation methods. The main feature of this selfcalibration technique is that it does not relate all the images in a single projective frame (which is hard to do in some cases), only pairwise epipolar calibration. The drawback of this technique is that it involves high computational costs and it is difficult to take into consideration the uncertainty associated with the estimate of the epipolar geometry and any a priori knowledge regarding the intrinsic parameters that might be available. Variants of the basic approach [1] were proposed by Heyden and Astrom [7].

Pollefeys et al. [8, 9] proposed to minimize the deviation from Equation (5) in a stratified approach, which starts from projective calibration, augments it with the homography of the plane at infinity to yield affine calibration and finally upgrades to Euclidean calibration. The method is developed further in [10] to obtain the metric calibration of a camera setup from only three images.

Hartley in [5] uses the ideas in [1] to develop a practical algorithm for self-calibration for more than three cameras. A global optimization technique is defined where a lot of smaller optimization problems have to be solved in order to get a starting point for the last optimization.

Triggs [11] propose to minimize the deviation from Equation (7) that is the recovery of the absolute quadric. The absolute quadric is a degenerate quadric consisting of planes tangent to the absolute conic and has the property that its image projection coincides with the dual image of the absolute conic. An initial projective reconstruction is 
rectified to give calibration and scaled Euclidean structure and motion. The absolute quadric and conic are recovered simultaneously using an efficient constrained nonlinear optimization technique or a quasi-linear method.

Lourakis and Deriche [12] propose a simplification of the Kruppa equations and show how it can be employed for self-calibration. The simplification is derived in a purely algebraic manner and is based solely on the fundamental matrix. Estimates of the epipoles, which are known to be difficult to compute accurately, are not needed. And hence, this algorithm has the potential of being more stable and robust with respect to measurement noise.

Lei et al. [13] propose a method to solving the Kruppa equations (Equation 6) for camera self-calibration. First, the method determines the scale factors by a LevenburgMarquardt (LM) optimization or Genetic optimization technique. Then the camera's intrinsic parameters are derived from the resulting linear constraints.

\subsection{Varying intrinsic}

Heyden and Astrom [14] have shown that selfcalibration in the case of continuously focusing/zooming cameras is possible when the aspect ratio is known and no skew is present. However, although their method estimates intrinsic calibration through a non-linear bundle adjustment algorithm, no means of obtaining a suitable initial solution was proposed. Extending their work, Pollefeys et al $[15,16]$ prove that the absence of skew in the image plane is sufficient to allow for self-calibration. They also proposed an optimization framework for recovering the intrinsic parameters. Heyden and Astrom [17] have proven a more general result according to which the existence of one constant intrinsic parameter suffices for self-calibration. In all of the aforementioned methods [14-16], variable intrinsic calibration parameters are estimated through the recovery of the absolute quadric [11].

The first step in recovering the absolute quadric is to obtain a projective reconstruction that uses bundle adjustment to compute for each image a projection matrix that is consistent with inter-image correspondences and is known up to a $3 \mathrm{D}$ projective transformation. Then, the motion rigidity assumption is utilized in some constrained optimization framework to yield the intrinsic parameters and rectify the projective reconstruction to a Euclidean one. The problem of this iterative approach is that iteration is quite chancy in the context of self-calibration under minimum assumptions on internal parameters. Hartley et al. [18] replace the need for descent-based iteration by a quick, but exhaustive search for the best affine reconstruction.

Pollefeys et al. [19] use the modulus constraint where the self-calibration method presented in [8] is extended to allowing changing focal length. However, the practical implications of this result are questionable since when the focal length varies, by zooming, the principal point varies also. The method is developed further in [10]. They reduce Equation (5) to a set of linear equations by following a stratified approach. They first obtain the homographies of the plane at infinity by first reaching an affine calibration based on modulus constraint.

Hartley [20] Provide an algorithm for computing the focal lengths of two cameras given the corresponding fundamental matrix and knowledge of the remaining intrinsic parameters. That paper made the first use of the Huang and Faugeras constraints, presented in [21], as a tool for self-calibration. Bougnoux [22] provides an elegant closed-form solution for the same problem. He estimates the projective projection matrices corresponding to the available images and embeds them into a nonlinear minimization algorithm for finding the homography that maps the estimated projection matrices to Euclidean ones. The Kruppa equations are employed for obtaining an initial solution for the nonlinear minimization process.

Mendonca and Cipolla [23] exploit the well-known equality constraint regarding the two nonzero singular values of the essential matrix [20,21]. Since the latter is a function of the fundamental matrix and the unknown intrinsic parameters, the equality constraint corresponds to a constraint involving the intrinsic parameters that are recovered by optimizing an appropriate objective function. The method presented in [23] generalizes the ones in [20, 22]. It computes first the focal lengths using linear approach then refines this estimate and estimates the principle point using a non-linear approach. It takes into account how close to a critical motion for self-calibration (see [24-26]) the relative motion between any two pair of cameras in the sequence is, and "weights" the information provided by that particular pair accordingly. The input for the algorithm is only a set of fundamental matrices and it does not need projective bundle adjustment.

Lourakis and Deriche [27] extend their previous work for simplifying the Kruppa equations and using them for self-calibration in the case of constant intrinsic parameters [12] to the case of varying intrinsic calibration parameters. The intrinsic calibration parameters are recovered using a nonlinear minimization scheme that explicitly takes into consideration the uncertainty that is associated with the estimates of the employed fundamental matrices. For all developments, only pairwise fundamental matrices need to be estimated, thus the need for a projective reconstruction is relaxed.

Rousso and Shilat [28] present a method to compute the pose estimation and the calibration from three homographies between two images, without first computing a projective camera. Three homographies can be obtained directly from the trifocal tensor or from the fundamental matrix between the two images. 
Seo and Heyden [29] present an algorithm for making flexible calibration from the assumption of zero skew. The purpose of their algorithm is to obtain good initial estimates for a subsequent bundle adjustment method or to make fast approximate auto-calibration. The algorithm is based on an initial projective reconstruction, in the form of a sequence of camera matrices. The Euclidean reconstruction is obtained from an iterative scheme, where the intrinsic parameters and the absolute conic are estimated sequentially, using only linear operations in each iteration. In this method a new constraint, called the orthogonality constraint, is used to constrain the location of the absolute conic.

\section{Camera intrinsic and motion constraints}

Many researchers proposed specific self-calibration algorithms for restricted motion, combining camera motion constraints and camera intrinsic constraints. In several cases it turns out that simpler algorithms can be obtained. However, the price to pay is that the ambiguity can often not be restricted to metric. Some interesting approaches were proposed in [30-42].

\subsection{Constant intrinsic}

Moons et al [30] and Hartley [31] propose to reduce Equation (5) to a set of linear equations by following a stratified approach. They first obtain the homographies of the plane at infinity by first reaching an affine calibration based on: pure translation [30], pure rotation [31].

Armstrong et al. [32] consider the case of selfcalibration of a camera under planar motion. A planar motion consists of a translation in a plane and a rotation about an axis perpendicular to that plane. It is often performed by a vehicle moving on the ground. They introduce the pole/polar relation between the vanishing point of the rotation axes and the trifocal line.

Faugeras et al. [33] investigate the relationship between the usual 2D camera and the 1D camera. 1D projective camera projects a point in $\mathrm{P} 2$ to a point in P1. A laser beam can be modeled as 1D camera. They find out that a 2D camera undergoing planar motion can be reduced to a 1D camera on the trifocal plane of the $2 \mathrm{D}$ cameras. A linear algorithm is used for 1D camera self-calibration instead of solving complicated Kruppa equations for 2D camera self-calibration.

Wang et al. [34] analyze the errors introduced when the assumption of a pure rotation about the camera's optic is violated. They consider the case of the intrinsic parameters remaining unchanged throughout the sequence. They analyze un-calibrated camera in addition to partially calibrated cameras, incorporating conditions of known principle point, aspect ratio and/or skew. They consider random translations caused for instance by shaky handheld operation.

\subsection{Varying intrinsic}

De Agapito et al. [35, 36] propose a linear method for self-calibration of a stationary but rotating camera. The internal parameters of the camera are allowed to vary from image to image, allowing for zooming (change of focal length) and possible variation of the principal point of the camera. The method works under the minimal assumption of zero-skew (rectangular pixels), or the more restrictive but reasonable conditions of square pixels, known pixel aspect ratio, and known principal point. Being linear, the algorithm avoids the convergence problems characteristic of iterative algorithms. They use the same approach as [31] (reduce Equation (5) to a set of linear equations by following a stratified approach) but for a zooming camera. The approach does not apply to some cases such as fixed, but unknown aspect ratio or fixed but unknown principle point. The proposed method is developed further in [37].

Kim and Hong [38] adopt a pan-tilt camera (without zaxis rotation) and simplify the intrinsic parameters to overcome the degenerated configuration and get a closed form solution. They first calculate the rotation angles then the focal lengths.

Tordoff and Murray [39] address the problem of radial lens distortion showing strong effect of the distortion (specially barreling distortion) on the accuracy of the selfcalibration of a rotating camera. They propose a method for automatically estimating the radial distortion over a sequence of images when both distortion and camera internal parameters vary.

Seo and Hong [40] show that, when skew is assumed zero, self-calibration of a zooming and rotating camera is possible and unique up to a rotation. They propose an iterative estimation method. The method is developed further in [41]. They show that one inter-image homography is necessary for computing internal camera parameters assuming that aspect ratio and principle point are fixed in time. Practical problems are also discussed.

Hayman and Murray [42] develop expressions describing the errors introduced when the assumption of a pure rotation about the camera's optic center is violated. They consider the case where the rotation arm is of fixed length and the focal length is the sole unknown parameter but can vary from image to another. They reach to the conclusion that the assumption of pure rotation is a perfectly good one in many practical situations when the distance to the scene is large in comparison with the translations of the camera.

\section{Camera intrinsic and scene constraints}

Recently some methods were proposed to combine selfcalibration with scene constraints. Some interesting approaches were proposed in [43-56]. 


\subsection{Constant intrinsic}

Li et al. [43] propose a self-calibration method that can compute the 5 intrinsic parameters linearly. In this method, the planar information in the scene is used, and the camera undergoes $\mathrm{N}(\mathrm{N}>=2)$ sets of three mutually orthogonal motions or $\mathrm{N}(\mathrm{N}>=5)$ sets of two orthogonal planar motions.

Triggs [44] develops a self-calibration technique based on some constraints involving the absolute quadric and the scene-plane to image-plane collineations. Since only the collineations with respect to a reference view (a key image) can be used to self-calibrate a camera with constant internal parameters, inaccurate measurements or poor conditioning in the key image contribute to all the collineations reducing the numerical accuracy or the stability of the method. Malis and Cipolla [45, 46] propose to impose the constraints between collineation using a different iterative method. They do not use any key image but all the images are treated equally averaging the uncertainty over all of them.

Sturm and Maybank [47] and Zhang [48] independently proposed to use planar patterns in 3D space to precisely calibrate cameras. While Sturm and Maybank also discuss singularities, Zhang also calibrates radial distortions. They both require a planar pattern known a priori. Lowbowitz and Zisserman [49] describe a technique of metric rectification for perspective images of planes using metric information such as a known angle, two equal but unknown angles, or a known length ratio.

$\mathrm{Xu}$ et al. [50] propose a linear algorithm to solve the problem of self-calibrating cameras, and recovering camera motion and plane equations from knowing two homography matrices for two planes in space. They use estimates as an initial guess that is further optimized by minimizing the difference between observations and reprojections.

Bondyfalat and Bougnoux [51] proposed a method of elimination to impose the scene constraints. Liebowitz and Zisserman [52] on the other hand formulate both the scene constraints and the self-calibration constraints as constraints on the absolute conic so that a combined approach is achieved. Vanishing points of orthogonal directions and rectified planes are examples of the scene constraints.

\subsection{Varying intrinsic}

In [53], Malis and Cipolla extend their work in [45, 46] to allow the recovering of the varying focal length. $\mathrm{Lv}$ et al. [54] describe an approach for estimating both intrinsic and extrinsic parameters from three orthogonal vanishing points and an object of known height. The method derives a direct solution of the parameters without using matrix manipulations, which should result in numerical stability. One vertical vanishing point and a horizontal vanishing line provide an approximate solution with some assumptions.

Zhang and Schenk [55] propose a new method for obtaining camera calibration of a stereovision system over time without using again any particular calibration apparatus. The idea is to use previously valid camera projection matrices and image point matches to push forward the Euclidean structure of the scene, which allows recalibrating the stereovision system. Uncertainty is systematically manipulated and maintained.

Mendelsohn and Daniilidis [56] propose and evaluate a technical solution to decrease the sensitivity of selfcalibration by placing easily identifiable targets of known shape in the environment. The relative position of the targets need not be known a priori. Assuming an appropriate ratio of size to distance these targets resolve known ambiguities. Constraints on the target placement and the camera's motions are explored.

\section{Critical motion sequences}

In some cases the motion of the camera is not general enough to allow for self-calibration. This kind of motion is defined as critical motion sequences. In the case of a critical motion sequence, there is no unique solution for the self-calibration problem. Hence, one can find more than one solution for the intrinsic camera parameters that satisfy all constraints on the camera parameters for all images of the sequence.

Sturm $[25,26]$ provides a complete analysis of critical motion sequences for constant intrinsic parameters and identifies specific degeneracies for some algorithms. Partial analysis and some specific cases are mentioned in [57-59]. Kahl [24] provides an analysis for the critical motions in case of varying intrinsic parameters.

Sturm [60] presents a type of camera motion that is not critical for the generic self-calibration problem, but for which the Kruppa equation approach fails. This is the case if the optical centers of all cameras lie on a sphere and if the optical axes pass through the sphere's center.

\section{Conclusion}

The area of camera self-calibration has drawn the attention of tens of computer vision scientists and researches in the last 10 years following the break through paper [1]. In order to calibrate the camera on-line, researches have used the camera intrinsic constraints separately and in conjunction with the camera motion constraints or the scene constraints. Most of the selfcalibration algorithms are concerned with unknown but constant intrinsic camera parameters. Recently, camera self-calibration in the case of varying intrinsic camera parameters was also studied. A group of researchers have also analyzed the developed self-calibration algorithms, estimated the errors, and identified critical motion 
sequences that can prevent from having a unique solution for the self-calibration problem.

The trend of the research in the self-calibration area is to use the camera intrinsic constraints in conjunction with the camera motion and the scene constraints while taking into consideration the critical motion sequences and provide a closed-form solution that can be solved linearly. Yet, we have not seen a smart algorithm that is based on a data fusion approach when using the available constraints; camera intrinsic, motion and scene. That approach should enhance the robustness and widen the usage of the selfcalibration method.

\section{References}

[1] S. Maybank and O. D. Faugeras. "A theory of selfcalibration of a moving camera," Int. Journal of Computer Vision, 8(2), pp.123-151, August 1992.

[2] O. D. Faugeras, Q. T. Luong, and S. J. Maybank, "Camera self-calibration: Theory and experiments," In G. Sandini, editor, Proc. 2nd European Conf. On Comp Vision, Lecture Notes in Comp. Science 588, pp. 321-334. Springer-Verlag, May 1992.

[3] R. T. Azuma, "A survey of augmented reality," Presence, 6:355-385, 1997.

[4] E. Kruppa, "Zur ermittlung eines objektes aus zwei perspektiven mit innerer orientierung," Sitz.-Ber. Akad. Wiss., Wien, math. naturw. Abt. IIa, 122:1939-1948, 1913.

[5] R. Hartley, "Euclidean reconstruction from uncalibrated views," In J. L. Mundy, A. Zisserman, and D. Forsyth, editors, Applications of Invariance in Computer Vision, Lecture Notes in Computer Science 825, Springer-Verlag, pp. 237-256, 1994.

[6] Q. T. Luong and O. Faugeras, "Self-calibration of a moving camera from point correspondences and fundamental matrices," Int. Journal of Computer Vision, 22(3), pp. 261-289, 1997.

[7] A. Heyden and K. Astrom, "Euclidean Reconstruction from Constant Intrinsic Parameters" Proc. 13th Int. Conf. on Pattern Recognition, IEEE Computer Soc. Press, pp. 339-343, 1996.

[8] M. Pollefeys, L. Van Gool and A. Oosterlinck, "The Modulus Constraint: A New Constraint for Self-Calibration", Proc. 13th International Conference on Pattern Recognition, IEEE Computer Soc. Press, pp. 349-353, 1996.

[9] M. Pollefeys and L. Van Gool, "Self-calibration from the absolute conic on the plane at infinity", Proc. Computer Analysis of Images and Patterns, Lecture Notes in Computer Science, Vol. 1296, Springer-Verlag, pp. 175-182, 1997.

[10] M. Pollefeys and L. Van Gool, "Stratified self-calibration with the modulus constraint", IEEE Trans. PAMI, 21(8), pp. 707-724, August 1999.

[11] B. Triggs, "Autocalibration and the absolute quadric," In Proc. Conf. CVPR, Puerto Rico, pp. 609-614, June 1997.

[12] M. A. Lourakis and R. Deriche. "Camera self-calibration using the singular value decomposition of the fundamental matrix," In 4th Asian Conference on Computer Vision, volume I, Taipei, Taiwan, pp. 403-408, January 2000.
[13] C. Lei, F. Wu, Z. Hu, and H. T. Tsui, "A new approach to solving kruppa equations for camera self-calibration," Int. conf. on Pattern recognition, Québec City, 2002.

[14] A. Heyden and K. Astrom, "Euclidean Reconstruction from Image Sequences with Varying and Unknown Focal Length and Principal Point," Proc. IEEE CVPR, pp. 438-443, 1997.

[15] M. Pollefeys, R. Khoch, and L. Van Gool, "Self-calibration and metric reconstruction in spite of varying and unknown internal camera parameters," In Proc. 6th Int. Conf. on Computer Vision, Bombay, India, pp. 90-95, January 1998.

[16] M. Pollefeys, R. Koch, and L. Van Gool, "Self-calibration and metric reconstruction in spite of varying and unknown intrinsic camera parameters," Int. Journal of Computer Vision, 32(1), pp. 7-25, August 1999.

[17] A. Heyden and K. Astrom, "Minimal Conditions on Intrinsic Parameters for Euclidean Reconstruction", Asian Conference on Computer Vision, Hong Kong, 1998.

[18] R. I. Hartley, E. Hayman, L. de Agapito, and I. D. Reid, "Camera calibration and the search for infinity," In proc. $7^{\text {th }}$ Int. Conf. on Computer Vision, kerkyra, Greece, pp. 510-517, 1999.

[19] M. Pollefeys, L. Van Gool and M. Proesmans, "Euclidean 3D Reconstruction from Image Sequences with Variable Focal Lengths", ECCV'96, Lecture Notes in Computer Science, Vol. 1064, Springer-Verlag, pp. 31-42, 1996.

[20] R. Hartley, "Estimation of relative camera positions for uncalibrated cameras," In G. Sandini, editor, Proc. 2nd Europ. Conf. on Comp. Vision, Lect. Notes in Comp. S. 588, Santa Margherita, Italy, Springer-Verlag, pp. 579-587, May 1992.

[21] T. S. Huang and O. Faugeras, "Some properties of the matrix in two view motion estimation," IEEE Trans. Pattern Analysis and Machine Intel., 11(12):1310-1312, Dec. 1989.

[22] S. Bougnoux, "From projective to Euclidean space under any practical situation, a criticism of self-calibration," Proc. 6th Int. Conf. on Computer Vision, India, pp. 790-796, Jan. 1998.

[23] P. R. S. Mendonca and R. Cipolla, "A simple technique for self-calibration," In Proc. CVPR., vol. I, Fort Collins, Colorado, pp. 500-505, June 1999.

[24] F. Kahl, B. Triggs, and K. Astrom, "Critical motions for auto-calibration when some intrinsic parameters can vary," $J$. of Mathematical Image and Vision, 13(2), pp. 131-146, Oct.2000.

[25] P. Sturm, "Critical motion sequences for monocular selfcalibration and uncalibrated Euclidean reconstruction," In Proc. Conf. Computer Vision and Pattern Recognition, San Juan, Puerto Rico, pp. 1100-1105, June 1997.

[26] P. Sturm, "Critical motion sequences for the self-calibration of cameras and stereo systems with variable focal length," In T. Pridmore and D. Elliman, editors, Proc. British Machine Vision Conference, volume I, Nottingham, pp. 63-72, Sept. 1999.

[27] M. A. Lourakis and R. Deriche. Camera self-calibration using the Kruppa equations and the SVD of the fundamental matrix: The case of varying intrinsic parameters, Technical Report RR-3911, INRIA, France, March 2000. 
[28] B. Rousso and E. Shilat, "Varying focal length selfcalibration and pose estimation from two images," Proc. of IEEE Conf. on Computer Vision and Pattern Recognition, Santa Barnara, California, pp. 469-475, June 1998.

[29] Y. Seo and A. Heyden, "Auto-calibration from the orthogonality constraints," Proc. of the Int. Conf. on Pattern Recognition, Barcelona, Spain, pp. 1067-1072, 2000.

[30] T. Moons, L. Van Gool, M. Proesmans and E. Pauwels, "Affine reconstruction from perspective image pairs with a relative object-camera translation in between", IEEE Trans. PAMI, vol. 18, no.1, pp. 77-83, Jan. 1996.

[31] R. Hartley, "Self-calibration from multiple views with a rotating camera", Lecture Notes in Computer Science, Vol. 800801, Springer-Verlag, pp. 471-478, 1994

[32] M. Armstrong, A. Zisserman and R. Hartley, "Euclidean Reconstruction from Image Triplets", ECCV'96, Lect. Notes in Computer Science, Vol. 1064, Springer-Verlag, pp. 3-16, 1996.

[33] O. Faugeras, L. Quan and P. Sturm, "Self-Calibration of a 1D Projective Camera and Its Application to the Self-Calibration of a 2D Projective Camera", IEEE trans. PAMI, 22(10), pp. 1179-1185, Oct. 2000

[34] L. Wang, S.B. Kang, H. Y. Shum, and G. Xu, "Error analysis of pure rotation-based self-calibration," Proc $8^{\text {th }}$ Int. conf. on Computer Vision, Vancouver, Canada, pp. I: 464-471, July 2001.

[35] L. De Agapito, R. Hartley and E. Hayman, "Linear calibration of a rotating and zooming camera," Proc. IEEE CVPR, Fort Collins, Colorado, pp. 15-21, 1999.

[36] L. De Agapito, E. Hayman, and I. Reid, "Self-calibration of a rotating camera with varying intrinsic parameters," In Proc. $9^{\text {th }}$ British Machine Vision Conf., Southampton, pp. 105-114, 1998.

[37] L. De Agapito, E. Hayman, and L. Reid, "Self-calibration of rotating and zooming camera," Int. Journal of Computer Vision, 45(2), pp. 107-127, 2001

[38] H. Kim and K. S. Hong, "A practical self-calibration method of rotating and zooming cameras," IEEE Proc. on Vision, Image and Signal Proc., Vol. 148 (5), pp. 349-355, Oct. 2001.

[39] B. Tordoff and D. W. Murray, "Violating rotating camera geometry: the effect of radial distortion on self-calibration", Proc. of Int. Conf. on Pattern Recognition, pp. 1423-1427, 2000.

[40] Y. Seo and K. Hong, "Auto-calibration of a rotating and zooming camera," In Proc. of IAPR workshop on Machine Vision Applications, pages 17-19, Nov. 1998.

[41] Y. Seo and K. Hong, "About self-calibration of a rotating and zooming camera: Theory and practice," In Proc. $7^{\text {th }}$ Int. Conf. On Computer Vision, Kerkya, Greece, 1999.

[42] E. Hayman and D. W. Murray, The effects of translational misalignment in the self-calibration of rotating and zooming cameras, Oxford Eng. Library TR OUEL 2250/02, Sept. 2002.

[43] H. Li, H. Zhang, F. Wu and Z. Hu, "A new linear camera self-calibration technique," Proc. of the $5^{\text {th }}$ Asian Conf. on Computer Vision, Melbourne, Australia, Jan. 2002.
[44] W, Triggs, "Auto-calibration from planar scenes," In proc. $5^{\text {th }}$ European Conference on Computer Vision, Freiburg, Germany, pages 89-105, 1998.

[45] E. Malis and R. Cipolla, "Camera self-calibration from unknown planar structures enforcing the multiview constraints between collineations," IEEE Tran. on Pattern Analysis and Machine Intelligence, 24(9), pp. 1268-1272, Sept. 2002.

[46] E. Malis and R. Cipolla, "Multi-view constraints between collineations: application to self-calibration from unknown planar structures," Proc. of European Conf on Computer Vision, pp. $610-624,2000$

[47] P. Sturm and S. Maybank, "On plane based camera calibration: a general algorithm, singularities, applications," In Proc. IEEE Conf. CVPR, pp. 432-437, 1999.

[48] Z. Zhang, "A flexible new technique for camera calibration," IEEE Trans. PAMI, 22(11), pp. 1330-1334, 2000.

[49] D. Liebowitz and A. Zisserman, "Metric rectification for perspective images of planes," Proc.CVPR, pp. 482-488, 1998.

[50] G. Xu, J. Terai and H. Shum, "A linear algorithm for Camera Self-Calibration, Motion and Structure Recovery for Multi-Planar Scenes from Two Perspective Images," Proc. of Conf. CVPR, Hilton Head Island, SC., pp. 474-479, June 2000.

[51] D. Bondyfalat and S. Bougnoux, "Imposing Euclidean Constraints During Self-Calibration Processes", Proc. SMILE Workshop (post-ECCV'98), Lecture Notes in Computer Science, Vol. 1506, Springer-Verlag, pp.224-235, 1998.

[52] D. Liebowitz and A. Zisserman, "Combining Scene and Auto-calibration Constraints", Proc. ICCV, pp.293-300, 1999.

[53] E. Malis and R. Cipolla, "Self-calibration of zooming cameras observing an unknown planar structure," Proc. of the Int. Conf. on Pattern Recognition, 2000.

[54] F. Lv, T. Zhao and R. Nevatia, "Self-calibration of a camera from video of a walking human," Proc. of the Int. Conf. on Pattern Recognition, 2002.

[55] Z. Zhang and V. Schenk, "Self-maintaining camera calibration over time," Proc. of Conf. on CVPR, 1997.

[56] J. Mendelsohn and K. Daniilidis, "Constrained selfcalibration,” Proc. IEEE Int. CVPR, pp. 581-587, 1999

[57] M. Pollefeys, Self-calibration and metric $3 D$ reconstruction from uncalibrated image sequences, PhD, K.U.Leuven, 1999.

[58] Y. Ma, S. Soatto, J, Kojsecka and S. Sastry, "Euclidean Reconstruction and Reprojection Up to Subgroups", Proc. ICCV, pp.773-780, 1999.

[59] F. Kahl, "Critical Motions and Ambiguous Euclidean Reconstructions in Auto-Calibration”, Proc. ICCV, pp. 469-475, 1999.

[60] P. Sturm, “A case against Kruppa's equations for camera self-calibration," IEEE Trans. PAMI, pp. 1199-1204, Oct. 2000. 